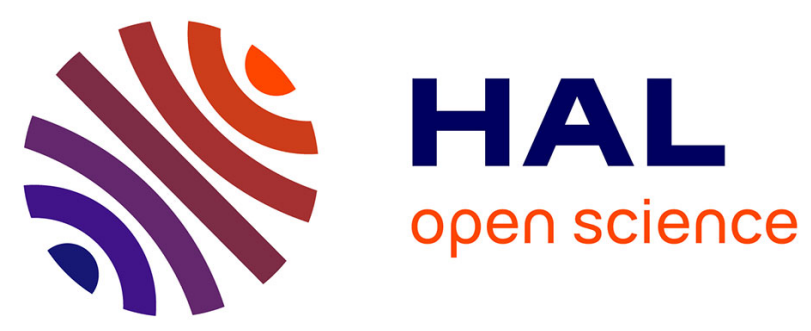

\title{
Time Undersampled Acquisition for Multidimensional Sparse Signals With Application to Magnetic Resonance Spectroscopic Imaging
}

Jabrane Karkouri, Helene Ratiney, Magalie Viallon, Remy Prost, Fabien Millioz

\section{To cite this version:}

Jabrane Karkouri, Helene Ratiney, Magalie Viallon, Remy Prost, Fabien Millioz. Time Undersampled Acquisition for Multidimensional Sparse Signals With Application to Magnetic Resonance Spectroscopic Imaging. IEEE Transactions on Signal Processing, 2021, 69, pp.5289-5298. 10.1109/TSP.2021.3112931 . hal-03370854

\section{HAL Id: hal-03370854 \\ https://hal.science/hal-03370854}

Submitted on 18 Oct 2021

HAL is a multi-disciplinary open access archive for the deposit and dissemination of scientific research documents, whether they are published or not. The documents may come from teaching and research institutions in France or abroad, or from public or private research centers.
L'archive ouverte pluridisciplinaire HAL, est destinée au dépôt et à la diffusion de documents scientifiques de niveau recherche, publiés ou non, émanant des établissements d'enseignement et de recherche français ou étrangers, des laboratoires publics ou privés. 


\title{
TIME UNDERSAMPLED ACQUISITION FOR MULTIDIMENSIONAL SPARSE SIGNALS WITH APPLICATION TO MAGNETIC RESONANCE SPECTROSCOPIC IMAGING
}

\author{
Jabrane Karkouri ${ }^{1,2}$, Hélène Ratiney ${ }^{1}$, Magalie Viallon ${ }^{1}$, Rémy Prost ${ }^{1}$, Life Member IEEE, Fabien Millioz ${ }^{1}$ \\ ${ }^{1}$ Université de Lyon, INSA Lyon, Université Claude Bernard Lyon 1, UJM-Saint-Etienne \\ CNRS, Inserm, CREATIS UMR 5220, U1206, F-69621 LYON, France \\ ${ }^{2}$ Siemens Healthineers, 40 avenue des fruitiers, 93210 Saint-Denis, France
}

\begin{abstract}
This paper deals with acceleration of multidimensional signal acquisition. The signal is assumed to have multiple discrete spatial dimensions where each point is time varying. The one-dimensional Fourier transform of the time evolution of each point is assumed to have an a priori known bounded support. The Fourier transform of the spatial domain is divided into several partitions, each of which can be sequentially acquired over time. We propose a method for undersampling the time dimension that enables interleaving of the samples hence speeding up signal acquisition. This method, applied to realistic simulated magnetic resonance spectroscopic imaging data (MRSI), leads to a reduction in the acquisition time by a factor of three.
\end{abstract}

Index Terms - Irregular sampling, undersampling, sample selection, sensor placement, least-square, magnetic resonance spectroscopic imaging, spiral spectroscopic imaging

\section{INTRODUCTION}

Compressed Sensing (CS) [1], [2] introduced a new way to sample, with a lowered sampling rate compared to the Nyquist-Shannon criterion. The signal to be sampled has to be sparse in a given space so that CS can be applied. CS has already been used for several applications in medical imaging [3], digital imaging [4] and radar imaging [5]. It is based on three main steps: sparse representation, measurement encoding, and sparse signal reconstruction [6].

For a known sparse spectra support, it is possible to accelerate and simplify the reconstruction process. In [7], [8] the $l_{2}$ norm was used to reconstruct the signal with a known sparse spectra support using the least square (LS) overdetermined solution. In [7] optimal k-space sampling in MRSI for images with a limited region of support is investigated while in [8] a fast multidimensional NMR spectroscopy for sparse spectra is proposed. Compared to the orthogonal matching pursuit algorithm (OMP)[9], the LS solution corresponds to only the last step of this algorithm when all of the samples of the support are found [10]. In a noisy scenario, the reconstruction error depends on the choice of the acquired time samples, but selecting the optimal choice is combinatorial. Computationally efficient methods leading to good suboptimal solutions are required. The first proposed algorithm was the Sequential Backward Selection (SBS) algorithm [11], iteratively removing samples up to the desired number, minimizing the Mean Squared Error (MSE) at each iteration. The same authors proposed an equivalent constructive algorithm called the Sequential Forward Algorithm (SFS), [12], but it requires a full rank matrix to start with, and thus some already selected samples. This sample selection problem is equivalent to a sensor placement problem, and thus has several solving algorithms and methods, such as approximating the problem using a convex relaxation [13], [14], or greedy methods. The latter consists in selecting samples one by one by optimizing a proxy of ideal acquisition, such as the MSE [15], the worst case error variance [16], the volume of the confidence ellipsoid [17], or the frame potential [18]. An exhaustive bibliography is given in [19], with an extension to these greedy methods, by searching samples among a given number of groups. One can note that SBS is a backward version of the greedy method minimizing the MSE, while SFS is identical as soon as the number of selected samples leads to a full rank matrix.

In the present study, we tackle the problem of the design of a fast sampling strategy for multidimensional signal acquisition, with its application to simulated magnetic resonance spectroscopic imaging (MRSI) data [7]. Here we use the sample selection algorithm in a different way, and we push further the method to design a new sampling strategy taking advantage of the multidimensional characteristic. The first results from this study were presented in [20].

Magnetic resonance spectroscopic imaging (MRSI) signals consist of several spatially distributed one-dimensional spectra. As for magnetic resonance imaging, the acquisition is performed in a spatial two-dimensional spectrum domain called the $\mathrm{k}$-space, for which $\left(k_{x}, k_{y}\right)$ represents the wave numbers in each dimension. In MRSI, this k-space evolves in time according to an oscillatory function with an exponential decay. The oscillation frequencies are characteristic of the molecular content under analysis. A two-dimensional Fourier transform of the k-space is then necessary to spatially 
localize the biochemical information, and a one-dimensional Fourier transform along the time direction is needed to obtain the magnetic resonance spectra. The time domain and the $\mathrm{k}$-space domain are separable. At each $\left(k_{x}, k_{y}\right)$ point, the associated spectrum is bandlimited. In in vivo magnetic resonance spectroscopy, it is customary to consider that molecular resonance frequencies in living tissues [21], [22] and the expected 'full width at half maximum' (FWHM) of the resonating peaks are known a priori. Moreover, frequencies and FWHM are used as starting values and prior knowledge in magnetic resonance signal quantification [23]; here, these are used to define this limited band a priori, i.e, the spectral support.

For the purpose of generality of this work, we deal with a spatio-temporal signal model $y(U, n)$, where $U$ is a $K$ multidimensional space and $n$ is the discrete time dimension. It is assumed that $y(U, n)$ can be fully acquired by sampling it sequentially over different partitions of $U$.

For this particular MRSI application, $U$ is the k-space. Due to physical constraints, it is not possible to scan the whole joint spatial and temporal dimensions of the signal all at once. Indeed, at each scan, data points are sequentially acquired over time and over limited regions of the k-space. Given that the signal is transient, several scans are required to obtain the full $U$ space over time. As a result, the full and dense sampling of this signal is time consuming. This approach is similar to multiple measurement vector methods [24], [25], which represent an extension of the single measurement vector methods, with the difference being that the acquisitions are sequential and the spectral support is known.

Several fast k-space scanning or CS methods have been proposed for MRSI [26], [22]. The undersampling of the temporal dimension has never been proposed except for a CS scenario [27]. In the special case of sparse spectra, we show that subsampling the temporal dimension enables interleaving of the acquisitions of the partitions of $U$ of the signal $y(U, n)$. Therefore, it leads to a decrease in the number of scans, and a reduction of the acquisition time of the signal. We propose an algorithm that specifies the signal acquisition scheme.

In this work, our main contribution is:

- a method to sample a multidimensional signal in a multidimensional framework in order to accelerate the acquisition while minimizing the MSE;

Other contributions include:

- a group extension of SBS, and its comparison to the state-of-the-art of one-dimensonnal sensor placement;

- theorems required for our proposal;

- an application of the proposed method to a realistic MRSI simulation with performances evaluation.

This paper is organized as follows. In section 2, we first extend SBS to a group method and compare it with

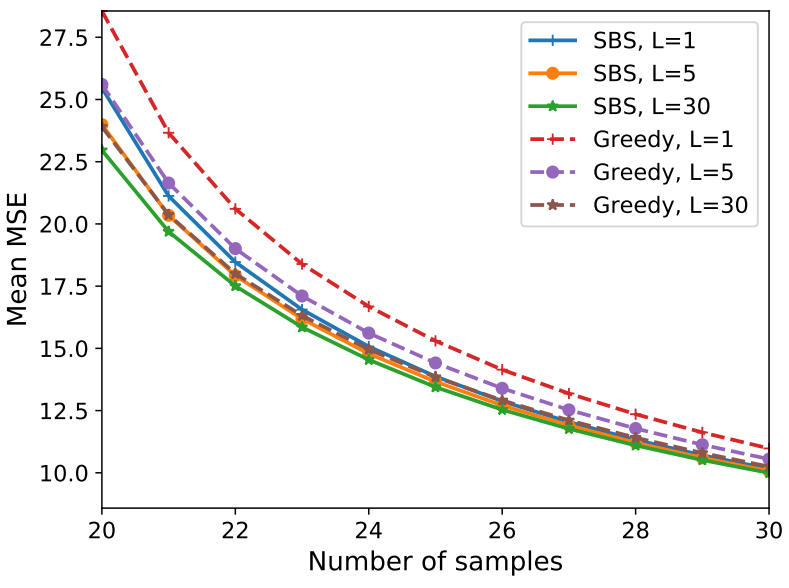

Fig. 1. MSE versus the sensor (or sample) number, for a $100 \times 20$ matrix $\Psi$, whose components follows a uniform distribution in $[0,1]$, for the group greedy method and the group SBS of size $L \in[1,5,30]$. MSE is averaged over 100 realizations.

a generic state-of-the-art method, and then apply it to a one dimensional sparse-spectrum signal to define an acquisition strategy. Finally two theorems are derived from this special case. Section 3 uses these theorems to propose a multidimensional signal acquisition algorithm. The MRSI application is presented in section 4 , followed by our conclusion and some perspectives.

\section{SAMPLE SELECTION FOR MSE MINIMIZATION}

\subsection{Problem statement and state-of-the-art}

We want to solve a linear inverse problem, expressed as

$$
y=\Psi x+\nu,
$$

where $y$ is a $N$-samples measurement vector, $x$ a $M$-samples unknown vector, $\Psi$ an $N \times M$ observation matrix, and $\nu$ a white Gaussian zero mean noise which is identically distributed, with variance $\sigma^{2}$.

We use $N$ measurements to estimate the $M$-dimensional $x$ vector, assuming $N>M$. In this case, the matrix system is overdetermined, and the minimum variance unbiased estimate of $x$ is the Least Square (LS) solution

$$
\hat{x}=\left(\Psi^{*} \Psi\right)^{-1} \Psi^{*} y,
$$

where ${ }^{*}$ denotes the conjugate transpose.

Given that (1) is overdetermined, it is possible to subsample $y$, by selecting only $P$ rows from $\Psi, M \leq P<N$.

This sample selection problem is strictly equivalent to a sensor placement problem: from the $N$ possible samples, which ones are the $P$ best leading to a minimal error?

Here we arbitrarily fix $P$ for targeted subsampling factor of $N / P$. The lower the subsampling, the higher the error. 
The choice of $\mathrm{P}$ is a tradeoff between subsampling and increased MSE. The optimal solution can be found by an exhaustive search, but this is computationally unaffordable. Algorithms leading to good solutions in a reasonable time, even if not optimal, are thus required.

Among these algorithms, greedy methods iteratively select rows of $\Psi$ one by one by optimizing a given criterion, arbitrarily chosen as the Mean Squared Error (MSE).

In this work, we use the MSE criterion as it is commonly used in MRSI.

The MSE of the estimate $\hat{x}$ of (2) is

$$
\operatorname{MSE}(\hat{x})=E\|x-\hat{x}\|^{2}=\sigma^{2} \operatorname{tr}\left[\left(\Psi^{*} \Psi\right)^{-1}\right] .
$$

The idea of the greedy method is to iteratively select the row of $\Psi$ that minimizes $M S E(\hat{x})$ at each iteration.

Note that when the number of selected rows is smaller than the number of columns $M, \Psi^{*} \Psi$ is singular. To avoid this problem, Jiang et al. [19] replace this singular matrix by $\Psi^{*} \Psi+\epsilon I$, where $\epsilon$ is a small number and $I$ the identity matrix. In addition, Jiang et al. extended the greedy method by searching for $L$ parallel groups at each iteration, with the intuition that it expands the solution search, and should be closer to optimal. They also compared their group greedy method [19] with five methods: FrameSense [18], convex relaxation, [13], SparSense [14], Greedy [16] [17] and even random selection, demonstrating that the group greedy method leads to the best results.

We propose here to expand the SBS method by using the group idea of [19], and compare it to the group greedy method using the same experiment.

The observation matrix $\Psi$ is a $100 \times 20$ uniform random matrix with independent components following a uniform distribution in $[0 ; 1]$. The greedy method and SBS are applied to this matrix, for groups sizes of $L \in[1,5,30]$, for a number of selected rows ranging from 20 to 30 . The MSE is computed for each case and averaged over 100 realizations of $\Psi$.

Fig. 1 shows the results. Overall, SBS gives better results than the greedy method. Moreover, as expected, the bigger the group, the lower the MSE, leading to a better sample selection.

\subsection{Application to sparse spectrum signals}

In this section, we consider a sparse spectrum signal

$$
y=F^{-1} x,
$$

where $y$ is an $N$-samples time-domain signal, with a sampling period $T_{s}, x$ its $N$-samples associated spectrum, and $F$ the unitary discrete Fourier matrix of size $N \times N$, $F_{n, k}=\exp (-i 2 \pi n k / N) / \sqrt{N}$.

Let $x_{m}$ be the restriction of $x$ to its $M$ non-zero values. Equation (4) becomes

$$
y=A x_{m}
$$

where $A$ is the $N \times M$ matrix that is obtained by selecting from $F^{-1}$ the columns that correspond to the $M$ elements of $x$ in its sparse support $S . A$ is the special case of $\Psi$ in (1).

We need to determine the best $P$ samples of $y$ to be acquired using a MSE-minimization algorithm, leading to a final $A$ matrix of size $P \times M$.

The SBS algorithm and the greedy method have been compared for group sizes of $L \in[1,5,30]$ with three types of support, which we define as compact, block, and random. Each of these support types has $M=128$ nonzero values and the total sample number $N=1024$. A compact support is defined as $M$ consecutive nonzero frequency locations. A block support is defined as eight different compact supports of size $M / 8$ that are randomly distributed. A random support is defined as $M$ randomly selected nonzero frequency locations.

Figure 2 shows the MSE evolution that is averaged on 50 random realizations of each support, versus the number $P$ of selected rows with $N=1024$. Several conclusions arise.

i) The more "compact" the support, the lower the MSE.

ii) Except for random support, using groups is harmful. When looking at the evolution of the MSE along the iterations, using groups shows better MSE at the beginning. However, selecting these better groups finally leads to higher MSE at the end. This shows the non-linearity of the iterative search. However, when looking at individual realizations, sometimes using large groups improves the MSE, while on average it does not.

iii) The greedy algorithm is on average better than the SBS one. Note that for certain realizations, SBS is better than the greedy method.

Based on these observations and given the particularities of the Fourier matrix, in the following, we choose to use as an MSE-minimization algorithm the greedy method, with a group size of 1 .

\subsection{Properties of the subsampled sparse Fourier matrix}

The following theorems give some properties of the matrix $A$ in (5) with only $P$ selected rows, which will be useful for our acquisition method described in section 3 .

\section{Theorem 1.}

Let $A$ be the $P \times M, M \leq P \leq N$, matrix constructed with the discrete Fourier transform matrix $F^{-1}$ of size $N \times N$ where $N-M$ columns and $N-P$ rows have been deleted. The entries of $A$ are $(A)_{n_{s}, k_{s}}=e^{2 \pi i n_{s} k_{s} / N} / \sqrt{N}$, where $k_{s}$ are the columns indexes corresponding to the support of $x$. The indexes $n_{s}$ are in the list $p$ of the $P$ selected rows in $F^{-1}$. Consider a circular shift $d$, with period $N$, of the indexes in $p$ and select the corresponding rows in $F^{-1}$ : if $A^{*} A$ is nonsingular, then $\operatorname{tr}\left[\left(A^{*} A\right)^{-1}\right]$ is invariant from this 

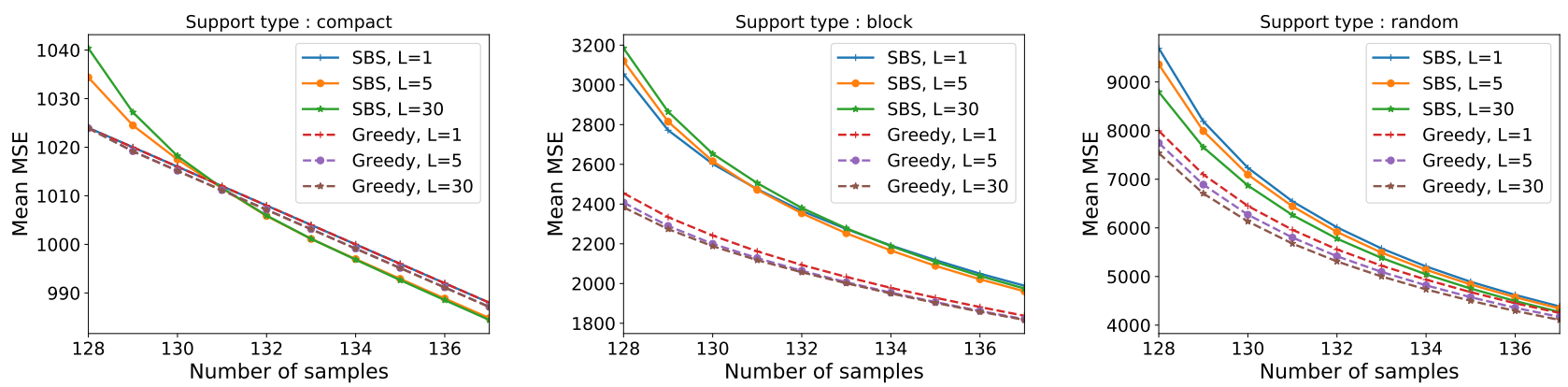

Fig. 2. MSE versus the sensor (or sample) number, for a Fourier matrix restricted to a random spectral support, for the group greedy method and the group SBS of size $L \in[1,5,30]$. Left: compact support. Center: block support. Right: random support. MSE is averaged over 50 realizations of each support.

circular shift.

\section{Proof.}

Consider a circular shift $d$ of the selected $P$ rows.

$$
\text { Write } \quad \begin{aligned}
\left(A^{d}\right)_{n_{s}, k_{s}} & =(A)_{n_{s}+d, k_{s}} \\
& =e^{2 \pi i\left(n_{s}+d\right) k_{s} / N} / \sqrt{N} .
\end{aligned}
$$

with $\left(n_{s}+d\right) \quad(\bmod N)$,

$$
\text { Then } \quad\left(A^{d}\right)_{n_{s}, k_{s}}=e^{2 \pi i d k_{s} / N}(A)_{n_{s}, k_{s}} .
$$

Then $\left(A^{d}\right)_{n_{s}, k_{s}}=e^{2 \pi i d k_{s} / N}(A)_{n_{s}, k_{s}}$.

It follows that

$$
A^{d}=A D^{d},
$$

where $D^{d}=\operatorname{diag}\left(e^{2 \pi i d_{s} k_{s} / N}\right), k_{s}=0,1, \ldots, M-1$.

Note that $D^{d *} D^{d}=I_{M}$, with $I_{M}$ the $M \times M$ identity matrix. Finally,

$$
\begin{aligned}
\operatorname{tr}\left[\left(A^{d *} A^{d}\right)^{-1}\right] & =\operatorname{tr}\left[\left(D^{d *} A^{*} A D^{d}\right)^{-1}\right] \\
& =\operatorname{tr}\left[\left(D^{d}\right)^{-1}\left(A^{*} A\right)^{-1}\left(D^{d}\right)^{-1 *}\right] \\
& =\operatorname{tr}\left[\left(D^{d *}\right)^{-1}\left(D^{d}\right)^{-1}\left(A^{*} A\right)^{-1}\right] \\
& =\operatorname{tr}\left[\left(A^{*} A\right)^{-1}\right] .
\end{aligned}
$$

\section{Involvement of Theorem 1}

For noisy data with the same hypothesis as in section 2.2, the selected time samples to be acquired can be circularly shifted, without increasing the resulting trace of the square matrix $\left(A^{*} A\right)^{-1}$, and therefore without increasing the noise amplification. This enables more flexibility in the choice of time samples used to solve (5) during the signal acquisition and this is a key point for our method based on the interleaved acquisition described in section 3.

\section{Theorem 2.}

Given the matrix A defined in Theorem 1, consider a circular shift $r$ of the support of $x$ with period $N$, the columns of the matrix $A$. If $A^{*} A$ is nonsingular, then $A^{*} A$ is invariant from the circular shift, and so is $\operatorname{tr}\left[\left(A^{*} A\right)^{-1}\right]$.
Proof.

Consider a circular shift $r$ of the $M$ selected columns.

Write $\left(A^{r}\right)_{n_{s}, k_{s}}=(A)_{n_{s}, k_{s}+r}$ $=e^{2 \pi i n_{s}\left(k_{s}+r\right) / N} / \sqrt{N}$.

Then $\left(A^{r}\right)_{n_{s}, k_{s}+r}=e^{2 \pi i r n_{s} / N}(A)_{n_{s}, k_{s}}$

It follows that

$$
A^{r}=D^{r} A
$$

where $D^{r}=\operatorname{diag}\left(e^{2 \pi i r n_{s} / N}\right), n=0,1, \ldots, P-1$.

Note that $D^{r *} D^{r}=I_{P}$, where $I_{P}$ is the $P \times P$ identity matrix.

Then $A^{r *} A^{r}=A^{*} D^{r *} D^{r} A=A^{*} A$

Involvement of Theorem 2

$\operatorname{tr}\left[\left(A^{*} A\right)^{-1}\right]$ is invariant from any circular shift of the spectrum support $(\bmod N)$, which does not require a new computation of the MSE minimization algorithm.

\section{MULTI-SETS ACQUISITION}

This section presents the signal model used in this study, and an algorithm whose goal is to minimize the acquisition time required to sample this signal.

\subsection{Model of the temporal signal $y(U, n)$}

Let $y(U, n)$ be a multidimensional signal, where $n$ is the discrete time dimension, and $U$ a $K$-dimensional space. $U$ is assumed to be divisable into $N_{u}$ partitions $U_{u}$, such that

$$
U=\cup_{u=0}^{N_{u}-1} U_{u} .
$$

Consequently, the signal $y(U, n)$ over all partitions is the union of each signal acquired over partitions

$$
y(U, n)=\cup_{u=0}^{N_{u}-1} y_{u}(n) \quad \forall n \in[0, N-1],
$$

where $y_{u}(n)$ is the signal acquired only on partition $U_{u}$.

At each $n$, it is assumed that only a single partition can be sensed, leading to a single $y_{u}(n)$. The acquisition of a 
single $y_{u}(n)$ over time is called time-scan in the following. $N_{u}$ time-scans of different signals $y_{u}(n)$ are required to collect the full signal $y(U, n)$ over of the $N_{u}$ partitions. For a uniformly sampled temporal dimension, the acquisition time is

$$
T_{a c q}^{f u l l}=T_{\text {scan }} N_{a c q}^{f u l l},
$$

where $T_{\text {scan }}=N T_{s}$ is the duration of one time-scan, $T_{s}$ is the sampling time period of the signal $y_{u}(n)$ and $N_{a c q}^{f u l l}=N_{u}$ the total number of time-scans.

To reduce the acquisition time, we subsample $y_{u}(n)$ to lower the number of data points along the temporal dimension. Each signal $y_{u}(n)$ is assumed to have an identical known sparse spectral support $S$. Thus, the signals $y_{u}(n)$ can be sub-sampled as described in section 2.2. Let $\{p\}$ be the set of all the selected time samples by the MSE-minimization algorithm with $\operatorname{Card}(\{p\})=P<N, P \geq M$.

As each $y_{u}(n)$ has the same spectral support $S$, it follows that the selected samples $\{p\}$ are also identical for each $y_{u}(n)$. At each time sample $p, N_{u}$ partitions have to be acquired simultaneously and thus $N_{u}$ time-scans are still required. To reduce the acquisition time, the idea is to take advantage of the unused time zones. The time indices $n \notin\{p\}$ will be used to acquire other partitions of $U$. From Theorem 1, these unused time zones can be filled by a circular shift of $\{p\}$.

The ideal scenario would be to fill the $N_{u} P$ samples to be acquired without any empty time zones during the $N$-points time-scan. This results in $N_{u} P / N$ time-scans compared to $N_{u}$ time-scans in the full sampled acquisition. The best acceleration factor is thus $N / P$. In practice, this is very unlikely to happen.

\subsection{Interleaving the acquisition in the time domain}

The objective is to minimize the total number of time-scans $N_{a c q}$ to acquire $y(U, n)$. This objective requires that the maximum number of partitions to be acquired for any $n$ should be minimal.

Let $\{p\}_{u}$ be the set of selected time samples for the signal of the partition $u, y_{u}(n)$. Let $I_{u}(n)$ be an index vector of length $N$ that indicates the $P$-samples to be acquired in partition $u, I_{u}(n)=1$ if $n \in\{p\}_{u}, 0$ otherwise. The total number of time-scans is then $N_{a c q}=\max _{n}\left(\sum_{u^{\prime}=1}^{N_{u}} I_{u^{\prime}}(n)\right)$.

The proposed algorithm minimizes the maximum number of partitions to be acquired for any $n$. It searches at each iteration $j$ for a set $\{p\}_{j}$ that minimizes the number of acquisitions considering only the first $j$ partitions,

$$
N_{a c q}^{j}=\max _{n}\left(\sum_{u^{\prime}=1}^{j} I_{u^{\prime}}(n)\right),
$$

where $j$ is from 1 to $N_{u}$.

The algorithm selects the best circular shift of $\{p\}$ that minimizes $N_{a c q}^{j}$, to determine $\{p\}_{j}$. In the case of multiple solutions, the smaller shift is selected. The first iteration sets $\{p\}_{1}=\{p\}$, and the final iteration acquire for each partition. Let us define $I_{1}(n-d \bmod N)$ as the $d$ circular shift of $I_{1}(n)$.

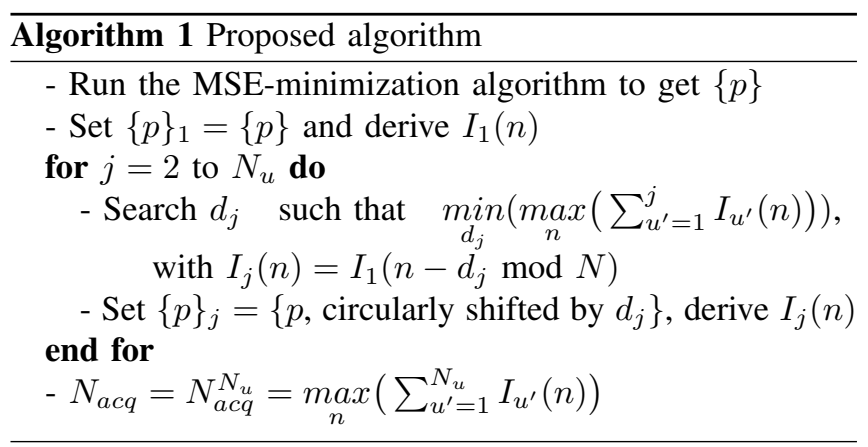

When the algorithm finishes, the $N_{u}$ set of points $\{p\}_{u}$ needed to acquire each partition are determined, as well as the number of acquisition required to do so.

The new acquisition time of $y(U, n)$ is then

$$
T_{a c q}=T_{s c a n} N_{a c q} .
$$

Compared to Equation (8), the time acceleration factor is $N_{\text {acq }}^{f u l l} / N_{a c q}=N_{u} / N_{a c q} \geq 1$.

\subsection{Illustration of the proposed algorithm}

An illustrative example of the proposed algorithm is used here, as a signal of size $N=16$ with a spectrum block support size $M=7$. The number of partitions is set to $N_{u}=3$. The MSE-minimization algorithm is run with $P=$ 8 time samples.

Figure 3 shows the iterative steps of the algorithm and the sets $\{p\}_{u}$ and $I_{u}(n)$ generated at each step. In this example, the final number of acquisitions $N_{a c q}$ is 2, to give a time acceleration factor of $3 / 2$. For each iteration, the new time samples are represented in red in Figure 3. In the first step, $\{p\}_{1}=\{p\}$ and $I_{1}(n)=1$ if $n \in\{p\}_{1}, 0$ otherwise. In the second step, there is no circular shift of $\{p\}_{1}$, such that the maximum of $I_{1}(n)+I_{2}(n)$ is 1 . In the third step, the algorithm finds a solution such that the maximum of $I_{1}(n)+$ $I_{2}(n)+I_{3}(n)$ leads to two time-scans.

Figure 4 illustrates how the partitions are acquired at each time-scan. During the first time-scan, it is always possible to entirely acquire the signal over the first partition (in blue), given that by definition a time-scan is the duration required to acquire the signal. Given than the signal over the first partition is subsampled, some time bins are unused. The algorithm shifts the selected samples to fill these holes for the other partitions. Consequently, 6 time samples related to the second partition and 2 time samples related to the third one are sampled during the first time scan. As a consequence, the remaining points of the last two partitions can be sampled in 


\begin{tabular}{|c|c|c|c|c|c|c|c|c|c|c|c|c|c|}
\hline \multirow{2}{*}{\multicolumn{2}{|c|}{$\begin{array}{c}M \\
N u\left(=N_{a c q}^{f u l l}\right) \\
\end{array}$}} & \multicolumn{4}{|c|}{64} & \multicolumn{4}{|c|}{128} & \multicolumn{4}{|c|}{256} \\
\hline & & 2 & 4 & 8 & 16 & 2 & 4 & 8 & 16 & 2 & 4 & 8 & 16 \\
\hline \multirow{3}{*}{$N_{a c q}$} & " compact support & $\overline{1}$ & 1 & $\overline{1} 1$ & 11 & $\overline{11}$ & $\overline{1}$ & $\overline{11}$ & 2 & $\overline{11}$ & $\overline{1} 1$ & 2 & $\overline{4}$ \\
\hline & block support & 1 & 1.0 & 1.6 & 2.3 & 1.0 & 1.5 & 2.4 & 4.5 & 1.6 & 2.4 & 5.2 & 11.5 \\
\hline & random support & 1 & 2.0 & 2.0 & 3.1 & 2 & 2.0 & 4.0 & 11.3 & 2 & 4 & 8 & 16 \\
\hline \multicolumn{2}{|c|}{ best $N_{a c q}=\left\lfloor N_{u} M / N\right\rfloor$} & 1 & 1 & 1 & 1 & 1 & 1 & 1 & 2 & 1 & 1 & 2 & 4 \\
\hline
\end{tabular}

Table 1. Given $N_{u}, M$ and different support types, the highest number of acquisitions $N_{a c q}^{f u l l}\left(=N_{u}\right)$, the lowest $N_{a c q}^{f u l l} M / N$ (rounded up) and the computed $N_{a c q}$ by the proposed algorithm are given, averaged over 100 realisations of the randomly chosen support. The greedy algorithm was used with $P=M$ and $N=1024$.
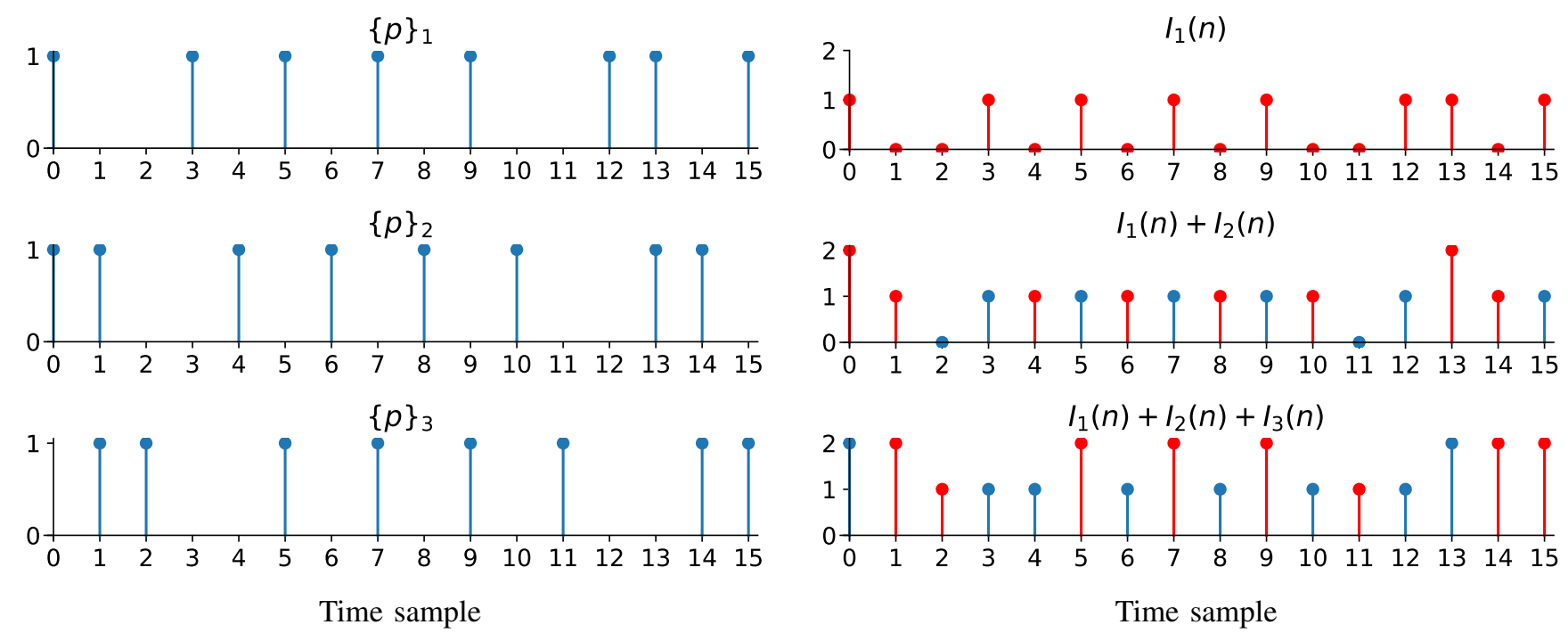

Fig. 3. Illustration of the proposed algorithm for $N=16, M=7$, and $P=8$. At each iteration, $\{p\}_{u}$ is a circular shift of $\{p\}_{1}$ (in this case, 1 shift for $\{p\}_{2}$ and 2 shift for $\left.\{p\}_{3}\right)$ which minimizes the maximum value of $\sum_{1}^{u} I_{u}(n)$. The maximum corresponds to the number of time-scans needed to sample all of the partitions.

a second time scan, avoiding the third time scan that would have been required in the case of an acquisition without interleaving.

\subsection{Study of the time acceleration factor}

To evaluate the time acceleration factor reached by the algorithm proposed in the previous section, the algorithm is run to test different spectral supports.

The signal size is set to $N=1024$; the spectrum has $M$ nonzero values, with $M \in\{64,128,256\}$; the support type of the spectrum is compact, block or random (See section 2.2); the number of partitions is $N_{u} \in\{2,4,8,16\}$.

The MSE-minimization algorithm is run with $P=M$ time points, which is the largest feasible subsampling with a given sparse spectrum.

For each set of parameters, the algorithm is run on 100 realizations of the randomly chosen support. For each run, the following are computed: the highest number of acquisitions required $N_{a c q}^{f u l l}=N_{u}$; the ideal lowest number of acquisitions $N_{a c q}^{f u l l} M / N$ (rounded up); and the number $N_{a c q}$ of acquisitions given by the proposed algorithm. The results are averaged over the 100 realizations, as in Table 1.
We can see that the acquisition speed-up, which is defined as $N_{a c q}^{f u l l} / N_{a c q}$, increases with the number of partitions and decreases with the number of samples in the spectrum support.

As expected, the sparser the spectrum, i.e., the lower the $M$, the lower the number of acquisitions. In comparison, the number of partitions $N_{u}$ has less influence. The support type has strong influence: the more "compact" it is, the better acquisition gain it gives. Interestingly, for a sparse spectrum of $M$ contiguous frequency samples, the algorithm always gives the lowest possible number of acquisitions.

\subsection{Performance and complexity of the algorithm}

The algorithm proposed in section 3.2 searches sequentially in $N_{u} \times N$ circular shifts for the combination of shifts that leads to the lowest number of time-scans. The total number of possible combinations is $N_{u}^{N}$. As seen in the previous section, this algorithm actually gives the lowest possible number of time-scans in the case of a compact support.

For the two other cases, an extensive search in the $N_{u}^{N}$ possibilities is run over 100 iterations of both the block and random supports, with a signal size $N=128$, 


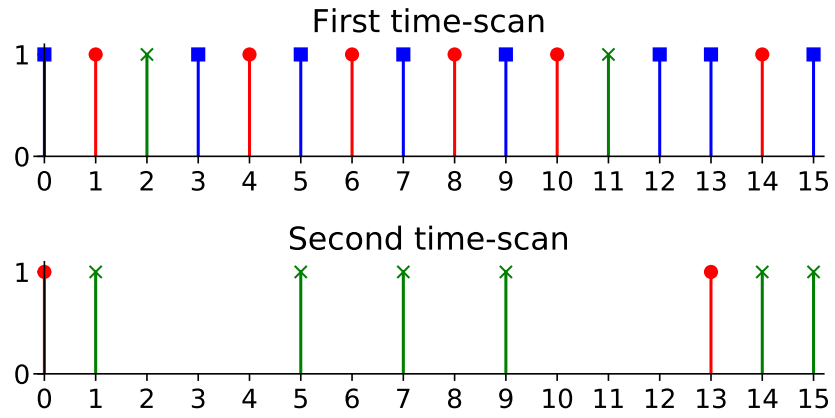

Time sample

Fig. 4. Each row corresponds to a time-scan of the algorithm illustrated in Figure 3. The first partition is in blue with a square symbol, the second is in red with a circle symbol, the third is in green with a cross symbol. Two time-scans are enough to sample all of the time samples of all of the partitions.

$N_{u}=4$ partitions and spectra of $M$ nonzero values, $M \in\{8,16,32,64\}$. When the spectrum is very sparse, $M=8$, many combinations lead to the best possible $N_{a c q}$, and the proposed algorithm always finds this. On the other hand, when half the spectrum points are used, $M=64$, the extensive search gets a better result than our algorithm in each run for a random support, and in 44 runs for a block support. For $M=16$ and $M=32$, the extensive search gets a better $N_{a c q}$ in $3 \%$ of the runs for a block support, and in $27.5 \%$ of the runs for a random support.

The proposed algorithm first runs the MSE-minimization algorithm, then performs circular shifts as detailed in section 3.2. The greedy MSE-minimization algorithm has a complexity of $O\left(N P M^{2}\right)$ [19]. The proposed algorithm in section 3.2 (excluding the MSE-minimization) has a complexity of $O\left(N^{2} N_{u}\right)$. The LS reconstruction has a complexity of $O\left(M^{2} P\right)$ for each partition. Note that the selection of the temporal samples to acquire (MSE-minimization) is used only once before the acquisitions.

\section{APPLICATION}

\subsection{Magnetic resonance spectroscopic imaging (MRSI)}

Nuclear magnetic resonance relies upon in a physical phenomenon that induces a perturbation of the macroscopic magnetization of an ensemble of nuclear spins that interact with a surrounding static magnetic field. This perturbation is caused by radiofrequency pulses, which are more commonly called excitation pulses, and lead to an electromagnetic signal response with frequencies related to the atoms considered and their chemical environment [22]. This signal is called the free induction decay and it is encoded in the k-space with the help of magnetic field gradients. It is complex, of the form $y\left(k_{x}, k_{y}, t\right)$, depending on the time $t$ and the spatial two-dimensional excited environment. In magnetic resonance imaging, the signal $y\left(k_{x}, k_{y}, t\right)$ is acquired in the Fourier domain, which is called the k-space excitation, at a given $t$. To obtain the corresponding anatomic image, an inverse Fourier transform is performed. In MRSI, we are also interested in the temporal evolution of the signal, which is why it is acquired for $t=n T_{s}, n \in[0,(N-1)]$. For each $\left(k_{x}, k_{y}\right)$, the signal is modeled by

$$
y_{k_{x}, k_{y}}(n)=\sum_{j} a_{j}\left(k_{x}, k_{y}\right) \exp \left(i 2 \pi f_{j} n T_{s}-n T_{s} / T_{2}^{*}\right),
$$

where the time constant $T_{2}^{*}$ is the transverse relaxation that accounts for the spin-spin relaxation and the field inhomogeneity. Due to the term $\exp \left(-n T_{s} / T_{2}^{*}\right)$, its spectrum is not strictly bandlimited because each spectral line becomes Lorentzian shaped; however, it can be approximately considered as sparse [8]. The coefficients $a_{j}\left(k_{x}, k_{y}\right)$ are complex and are the Fourier transform of the metabolic image characterized by the frequency $f_{j}$.

Each radiofrequency pulse excitation results in the same free induction decay signal. Excitations can be repeated as many times as necessary to get the joint spatial spectral information. At the end of the scan, a delay can be added to ensure that the macroscopic magnetization returns to its original state. The time between two excitations is called the repetition time, $T_{R} \geq N T_{s}$.

In routine clinical work, the sequences that are commonly used acquire the $k-t$ space, $\left(k_{x}, k_{y}\right)$-point by $\left(k_{x}, k_{y}\right)$-point, with the phase encoding method [26]. A single point of the $\mathrm{k}$-space and the whole temporal dimension is acquired at each excitation. This results indeed in a long acquisition time because, in that case, $N_{u}$, the number of partitions of the kspace, will be very large (see equation (8)). For example, for a $32 \times 32$ matrix, the number of $\left(k_{x}, k_{y}\right)$ points is $N_{u}=$ $32 \times 32=1024$. To speed up the acquisition, it is possible to group the k-space points into partitions, that have to be acquired after each excitation, which leads to the model of (6). However, due to technical constraints, the acquisition of one partition is not instantaneous. The larger the partition is, the longer the acquisition is. There is an issue if the partition acquisition time is longer than the sampling time period $T_{s}$, because this means that the next time sample is to be acquired while the previous one is still in the process of being acquired. Let $T_{\text {part }}$ be the acquisition time of each partition. The acquisition of each partition lasts for $N_{p}=$ $\left\lfloor\frac{T_{\text {part }}}{T_{s}}\right\rfloor$ time sampling periods and forbids the acquisition of the next $N_{p}-1$ time sample. $N_{p}-1$ additional excitations are required to acquire the full signal. This is called temporal interleaving. Moreover, as the repetition time $T_{R}$ is longer than $N T_{s}$, the total time acquisition time can be rewritten as

$$
T_{a c q}^{f u l l^{\prime}}=T_{R} N_{u} N_{p} .
$$

Acquiring the whole signal requires $N_{u} \times N_{p}$ excitations. 

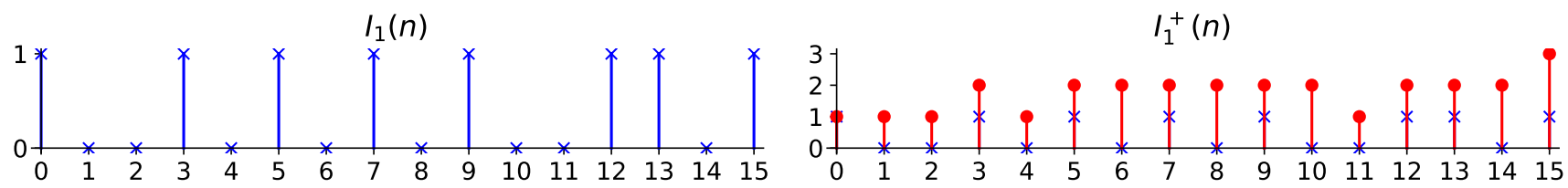

Fig. 5. Illustration of the difference between $I_{1}(n)$ and $I_{1}^{+}(n)=\sum_{d=0}^{N p-1} I_{1}(n-d)$ with $N_{p}=4$. The same parameters as in Figure 3 are used here. $I_{1}(n)$ is shown with a cross symbol on these two panels, and $I_{1}^{+}(n)$ with a circle symbol in the right panel. $I_{1}^{+}(n)$ shows the number of excitations required for each $n$.

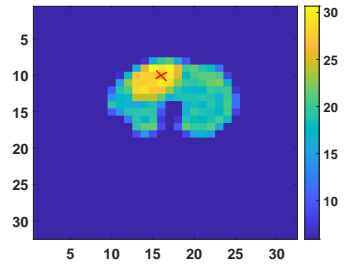

(a)

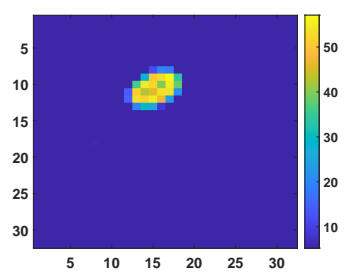

(c)

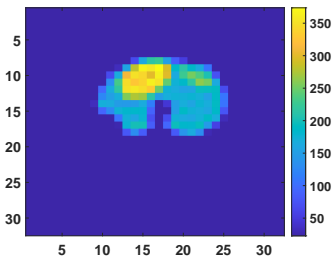

(b)

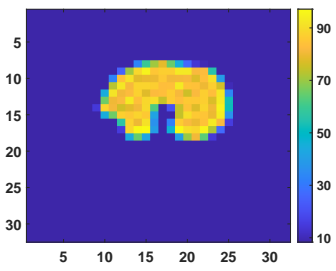

(d)
Fig. 6. a) Referenced phantom at $\mathrm{t}=0$ (based on ${ }^{31} \mathrm{P}$ in vivo image). Spectral images for (b) phosphocreatine, frequency = $0 \mathrm{~Hz}$, (c) inorganic phosphate, $\mathrm{f}=258 \mathrm{~Hz}$, and (d) $\gamma$ adenosine triphosphate, $\mathrm{f}=-129 \mathrm{~Hz}$. The red cross is used to locate the spectrum in Figure 8.

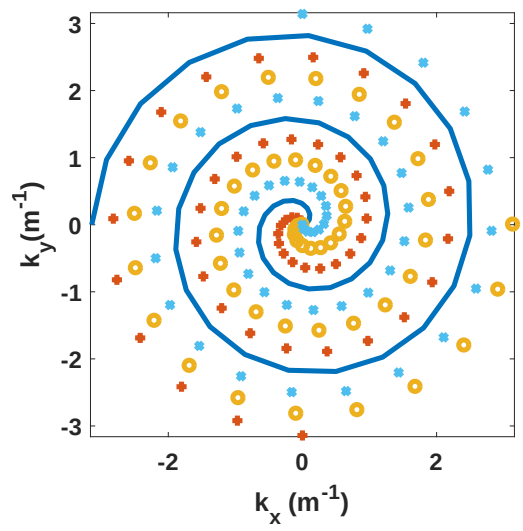

Fig. 7. Partitions of the k-space with $N_{u}=4$ spirals

Choosing small partitions, which are faster to sample, decreases $N_{p}$ but increases the number of partitions $N_{u}$ and vice versa.

\subsection{Time interleaved application to MRSI acquisition}

The additional constraint of the noninstantaneous acquisition of a partition requires an update of the proposed algorithm in section 3.2. In the previously proposed algorithm, the assumption of instantaneous acquisition of a partition is equivalent to $N_{p}=1 . I_{u}(n)$ is the index vector that indicates whether the time sample $n$ of the partition $u$ is to be acquired, consequently forbidding any other acquisition at time index $n$. With the constraint of the duration of one partition, a time sample at position $n$ forbids the $N_{p}-1$ following sample to be acquired. As an example for $N_{p}=2$, if there are two consecutive points to be acquired, the acquisition of the first one blocks out the acquisition of the second. This leads to at least two excitations to obtain the two points. By setting $I_{u}^{+}(n)=\sum_{d=0}^{N p-1} I_{u}(n-d)$, the number of excitations to acquire the partition $u$ is $\max \left(I_{u}^{+}(n)\right)$, as illustrated in Figure 5.

It follows then that the algorithm in section 3.2 requires the replacement of $I_{u}(n)$ by $I_{u}^{+}(n)$. To promote the scan of the beginning of the free induction decay where the signal magnitude is maximal, the $\{p\}_{u}$ are circularly left shifted, if necessary, using Theorem 1.

The steps to fully reconstruct the spectroscopic image $y(x, y, f)$ are the following:

- determine the time samples $\{p\}_{u}$ for each partition, through the algorithm in section 3.2 with $I_{u}^{+}(n)$ instead of $I_{u}(n)$;

- acquire each partition $y_{u}(n)$ for $n \in\{p\}_{u}$ of the kspace;

- apply LS reconstruction on each partition to obtain $y_{u}(f)$ (Equation (3));

- merge all partitions to get the whole $k-f$ space: $y\left(k_{x}, k_{y}, f\right)=\cup_{u=0}^{N_{u}-1} y_{u}(f)$;

- apply a two-dimensional nonuniform discrete Fourier transform [28], [29] to reconstruct the spectroscopic image $y(x, y, f)$.

Note that reproduction of the experiments of section 3.4 with the additional parameter $N_{p}$ shows that $N_{p}$ has similar influence to $N_{u}$ on the speed-up gain.

\subsection{Simulation and Results}

To compare the fully sampled method with the proposed interleaved time undersampling method, we use a numerical 
phantom of ${ }^{31} P$ spiral MRSI data, mimicking acquisition of the thigh muscle, with three compartments as defined in Figure 6-a and in which different metabolite ratios are used. The acquisition is simulated with a field of view of $25 \mathrm{~cm}$, and an image matrix size of $32 \times 32$. The k-space is partitioned in both methods with four spirals, as illustrated in Figure 6. The simulation parameters are $N_{u}=4$ partitions, time sampling period $T_{s}=0.25 \mathrm{~ms}, N=1024$ time samples, repetition time $T_{R}=2 \mathrm{~s}$, and spiral of $N_{p}=10$ discrete time samples which corresponds to a duration of $T_{\text {part }}=2.5 \mathrm{~ms}$. Each partition is a discrete spiral of 2048 points, which leads to 8192 points to sample the whole kspace. The support of the phosphorus spectrum contains two blocks, of total size $M=217$ samples. Here, the support is chosen such that the truncation of the spectral peaks is less than $1 \%$ of the total peak energy. In practice, the resonance frequency of the molecule is well known and the full width at half maximum depends on the static magnetic field homogeneity which is rather reproducible, so a support can always be determined a priori. For the proposed interleaved method, an objective of a best acceleration factor of 4 is used by setting $P=256$. As the spiral is not instantaneous in practice, every sample of the spiral is phase-corrected according to the Fourier shift theorem.

For the fully sampled spiral method, the acquisition time is $T_{a c q}^{f u l l^{\prime}}=80 \mathrm{~s}$. When applying the algorithm of section 4.2, this results in an acquisition time $T_{a c q}=26 \mathrm{~s}$, corresponding to a reduction by a factor of 3.08 .

The two methods are also compared in terms of the signalto-reconstruction error ratio (SRER), defined as

$$
\operatorname{SRER}=10 \times \log \frac{E\|x\|^{2}}{E\left\|x-x_{m}\right\|^{2}},
$$

with $x$ the original spectrum and $x_{m}$ the reconstructed one, both restricted to the spectral support.

Note that $x$, the original spectrum, is unknown. Incorporating (3) into (13), it follows

$$
\mathrm{SRER}=\mathrm{SNR}-10 \times \log \left(\operatorname{tr}\left[\left(A^{*} A\right)^{-1}\right]\right),
$$

where SNR is the unknown signal-to-noise ratio

$$
\mathrm{SNR}=10 \times \log \frac{E\|x\|^{2}}{\sigma^{2}} .
$$

Only the full sampled spectrum $x_{f}$ can be known. Its LS reconstruction is given by all the $N$ rows of the matrix $A$. The reconstruction error follows

$$
E\left\|x-x_{f}\right\|^{2}=\sigma^{2} M
$$

leading to the SRER for full sampling results

$$
\text { SRER_f }=\text { SNR }-10 \times \log \left(\sigma^{2} M\right) .
$$

The difference between the SRER_f and the SRER cancels the unknown SNR

$$
\text { SRER_f }- \text { SRER }=10 \times \log \left(\frac{\operatorname{tr}\left[\left(A^{*} A\right)^{-1}\right]}{M}\right) .
$$

In our experiments, the obtained undersampling scheme leads to SRER_f - SRER $=7.5 d B$.

\section{SRER performance for a realistic simulation}

Noisy spiral spectroscopic imaging data sets were generated, for both full sampling and subsampling with the proposed time interleaved acquisition, by adding complex white Gaussian noise samples to $y(U, n)$ (in the k-space, for all the partitions). The noise standard deviations were chosen to get two $S N R s$ : high SNR $(\approx 27 \mathrm{~dB}$ on average over all the samples containing signal energy), and low SNR $(\approx 18 \mathrm{~dB}$, on average). 50 noisy data sets per SNR were drawn and SRER as well as SRER_f were computed for each set, and SRER performance were analyzed on average over all 50 sets. The $T_{2}^{*}$ used for the exponential decay of the free induction decay signal was $100 \mathrm{~ms}$.

The results for the generated phosphorus numerical phantom of the thigh are shown in figures $6(\mathrm{~b}, \mathrm{c}, \mathrm{d})$ and 8 and table 2. Figure 6 shows the reconstructed phantom with the use of spiral spatial sampling, full time sampling, $S N R=27.38 \mathrm{~dB}$ for different molecular frequencies (i.e. (b) phosphocreatine $(\mathrm{PCr})$ for $\mathrm{f}=0 \mathrm{~Hz},(\mathrm{c})$ inorganic phosphate (Pi) for $\mathrm{f}=258 \mathrm{~Hz}$ and (d) $\gamma$-adenosine triphosphate $(\gamma$ ATP) for $\mathrm{f}=-129 \mathrm{~Hz}$ ). Figure 8 shows the reconstructed spectra at the location indicated by the red cross on Fig. 6, with a noisy acquisition for the fully sampling method and for the sub-sampling proposed method, with their respective errors.

In table 2, results over all the voxels containing signals and results restricted to the left ellipse of the signal from Fig. 7a) (where all molecules are present, and thus with a higher SNR with respect to the other regions) are given. The average of the SRER_f and SRER are computed over the 50 noisy sets and are given over two selected region: "Ellipse", the voxels in the left ellipse, and "All voxels", all the voxels with non-zero signal, plus or minus their associated mean standard deviation. One can see that in the noisiest scenario $(\mathrm{SNR}=18.67 \pm 2.61 \mathrm{~dB}$ ), the theoretical SRER difference between full and undersampled case (Equation 18) is recovered. With a higher SNR, the difference is higher than expected: the choice of the spectral support, which truncates the spectra that are not perfectly sparse, leads to an additional error reconstruction compared to the fully sampled case.

\section{CONCLUSION \& PERSPECTIVES}

A new acquisition method of interleaved time samples in undersampled signals is proposed here to speed up acquisition times, assuming a sparse known support.

Undersampling such a monodimensional signal can be done by minimizing the MSE of the LS reconstruction. We show that a circular shift of the selected samples does not change the reconstruction error. For multidimensional 

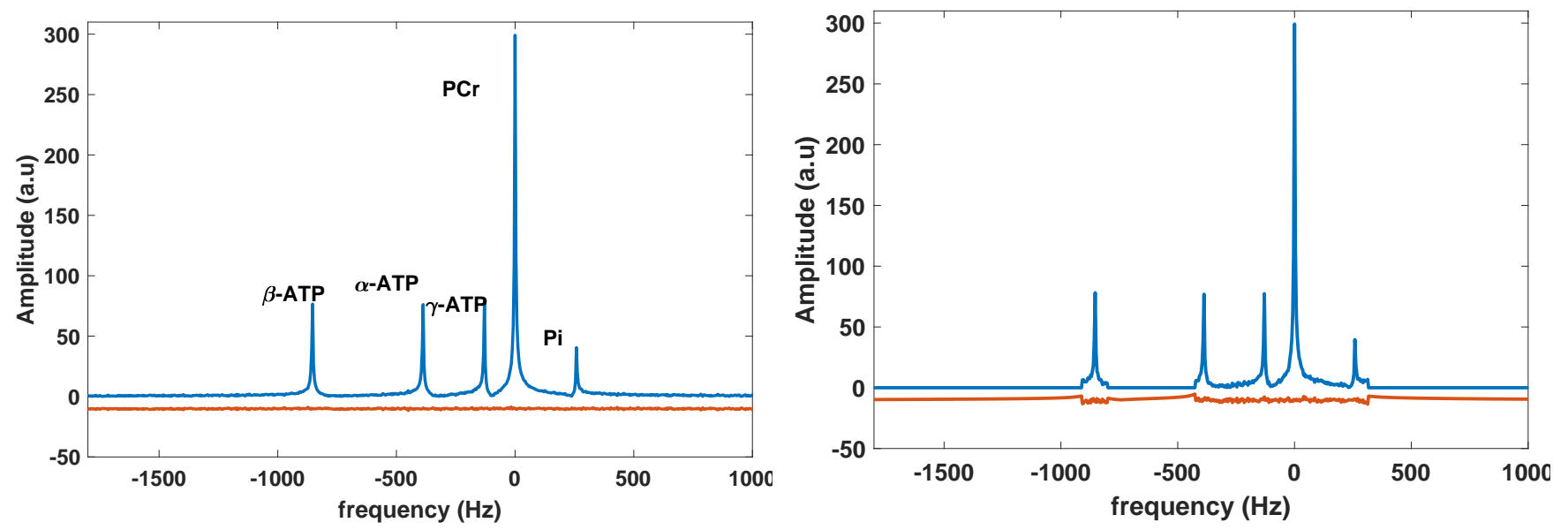

Fig. 8. Noisy reconstructed modulus spectrum from a voxel in the left ellipse (red cross in Figure 6-b) for the fully sampled spiral method (left) and for the new proposed approach (right). The reconstruction error (shifted by -10) is given at the bottom.

\begin{tabular}{|c||c|c||c|c|}
\hline & Ellipse & All voxels & Ellipse & All voxels \\
\hline SNR (dB) & $20.81 \pm 0.83$ & $18.67 \pm 2.61$ & $29.56 \pm 0.85$ & $27.38 \pm 2.61$ \\
\hline SRER_f (dB) & $18.25 \pm 1.69$ & $13.9 \pm 5.24$ & $35.76 \pm 1.7$ & $31.338 \pm 5.30$ \\
\hline SRER (dB) & $10.76 \pm 1.67$ & $6,5 \pm 5.25$ & $27.34 \pm 1.52$ & $23.35 \pm 5.08$ \\
\hline$S R E R_{f}-S R E R(\mathrm{~dB})$ & 7.49 & 7.4 & 8.42 & 8.04 \\
\hline
\end{tabular}

Table 2. SRER performances for noisy phantoms of phosphorus spiral spectroscopic imaging, described in Fig 6. Two standard deviations of noise were chosen, resulting in different averaged SNR according to the spatial regions analyzed ("Ellipse", voxels containing all molecules responses, "All voxels", any voxel containing nonzero signal).

signals, it means that different equivalent sets of samples can be selected for each additional dimension. By interleaving the partition sample acquisitions in the time domain, we can then reduce the total acquisition time. The proposed algorithm sequentially searches for the circular shift of the undersampling for each partition that will minimize the acquisition time. The proposed algorithm is applied here to MRSI. Our MRSI acquisition and reconstruction solution was compared to the results of a standard fully sampled spiral acquisition method which is here taken as the state-of-the art method. Note that among the accelerated sampling schemes [30], the spiral ones are the most efficient trajectories, offering the best acquisition time and spectral bandwidth benefit with the smallest SNR loss, but they are limited by a susceptibility to gradient infidelities. The results demonstrated that the acquisition time is reduced by a factor of 3.08 but the signal error SRER decreases by $7.5 \mathrm{~dB}$ in a realistic noisy acquisition.

The proposed interleaved acquisition strategy can, in theory, be generalized to three dimensional data (3D), with 3D partitioning, and (any) 3D trajectories that would regularly return to the center of $\mathrm{k}$-space but that would also require to be interleaved in the spectroscopic time dimension to reach a desired spectral bandwidth.

Other reconstruction algorithms that require knowledge of the support have been proposed in the literature and are interesting in terms of reconstruction performance compared to the $L_{2}$ reconstruction when the SNR deteriorates. We mention in particular the Approximate Message Passing for multiple measurement vector method [31] or the modified CS-based method for problems with partially known support proposed in [32].

A future goal will be to apply this method to exercise dynamics ${ }^{31} \mathrm{P}$ applications, accurate fat/water quantification, and ${ }^{1} \mathrm{H}$ MRSI or hyperpolarized ${ }^{13} \mathrm{C}$ studies. Magnetoencephalography can also be considered here, as an inverse problem used for brain imaging [33], [34]. Magnetoencephalography signals come from activated regions in the brain. Taking several signals over time enables the magnetic response of the brain to be followed over time. The activated regions could be known a priori based on the knowledge of brain functional structure, and our method can be applied.

\section{ACKNOWLEDGEMENTS}

This study was partly supported by the LABEX PRIMES (ANR-11-LABX-0063), program "Investissements d'Avenir" (ANR-11-IDEX-0007) and Siemens Healthineers. 


\section{REFERENCES}

[1] E. J. Candès, J. Romberg, and T. Tao, "Robust uncertainty principles: Exact signal reconstruction from highly incomplete frequency information," IEEE Transactions on information theory, vol. 52, no. 2, pp. 489-509, 2006.

[2] D. L. Donoho, "Compressed sensing," IEEE Transactions on information theory, vol. 52, no. 4, pp. 1289-1306, 2006.

[3] M. Lustig, D. L. Donoho, J. M. Santos, and J. M. Pauly, "Compressed sensing mri," IEEE signal processing magazine, vol. 25, no. 2, pp. 72-82, 2008.

[4] M. F. Duarte, M. A. Davenport, D. Takhar, J. N. Laska, T. Sun, K. F. Kelly, and R. G. Baraniuk, "Single-pixel imaging via compressive sampling," IEEE signal processing magazine, vol. 25, no. 2, pp. 83-91, 2008.

[5] M. A. Herman and T. Strohmer, "High-resolution radar via compressed sensing," IEEE transactions on signal processing, vol. 57, no. 6, pp. 2275-2284, 2009.

[6] E. J. Candès and M. B. Wakin, "An introduction to compressive sampling," IEEE signal processing magazine, vol. 25, no. 2, pp. 21-30, 2008.

[7] Y. Gao and S. J. Reeves, "Optimal k-space sampling in MRSI for images with a limited region of support," IEEE transactions on medical imaging, vol. 19, no. 12, pp. 1168$1178,2000$.

[8] D. Merhej, H. Ratiney, C. Diab, M. Khalil, M. Sdika, and R. Prost, "Fast multidimensional NMR spectroscopy for sparse spectra," NMR in Biomedicine, vol. 27, no. 6, pp. 640-655, 2014.

[9] J. Wang, S. Kwon, and B. Shim, "Generalized orthogonal matching pursuit," IEEE Transactions on signal processing, vol. 60 , no. 12 , pp. 6202-6216, 2012.

[10] J. A. Tropp and A. C. Gilbert, "Signal recovery from random measurements via orthogonal matching pursuit," IEEE Transactions on information theory, vol. 53, no. 12, pp. 4655-4666, 2007.

[11] S. J. Reeves and L. P. Heck, "Selection of observations in signal reconstruction," IEEE Transactions on Signal Processing, vol. 43, no. 3, pp. 788-791, 1995.

[12] S. J. Reeves and Z. Zhe, "Sequential algorithms for observation selection," IEEE Transactions on Signal Processing, vol. 47, no. 1, pp. 123-132, 1999.

[13] S. Joshi and S. Boyd, "Sensor selection via convex optimization," IEEE Transactions on Signal Processing, vol. 57, no. 2, pp. 451-462, 2009.

[14] H. Jamali-Rad, A. Simonetto, X. Ma, and G. Leus, "Distributed sparsity-aware sensor selection," IEEE Transactions on Signal Processing, vol. 63, no. 22, pp. 5951-5964, 2015.

[15] C. Jiang, Y. C. Soh, and H. Li, "Sensor placement by maximal projection on minimum eigenspace for linear inverse problems," IEEE Transactions on Signal Processing, vol. 64, no. 21, pp. 5595-5610, 2016.

[16] C. Jiang, Y. Soh, and H. Li, "Sensor and cfd data fusion for airflow field estimation," Applied Thermal Engineering, vol. 92, pp. 149-161, 2015.

[17] M. Shamaiah, S. Banerjee, and H. Vikalo, "Greedy sensor selection: Leveraging submodularity," in 49th IEEE Conference on Decision and Control (CDC), 2010, pp. 2572-2577.

[18] J. Ranieri, A. Chebira, and M. Vetterli, "Near-optimal sensor placement for linear inverse problems," IEEE Transactions on Signal Processing, vol. 62, no. 5, pp. 1135-1146, 2014.

[19] C. Jiang, Z. Chen, R. Su, and Y. C. Soh, "Group greedy method for sensor placement," IEEE Transactions on Signal Processing, vol. 67, no. 9, pp. 2249-2262, 2019.

[20] J. Karkouri, F. Millioz, M. Viallon, R. Prost, and H. Ratiney,
"Time samples selection in spiral acquisition for sparse magnetic resonance spectroscopic imaging," in Image Processing (ICIP), 2017 IEEE International Conference on. IEEE, 2017, pp. 4128-4131.

[21] V. Govindaraju, K. Young, and A. A. Maudsley, "Proton nmr chemical shifts and coupling constants for brain metabolites," NMR in Biomedicine, vol. 13, no. 3, pp. 129-153, 2000.

[22] R. A. De Graaf, In vivo NMR spectroscopy: principles and techniques, John Wiley \& Sons, 2019.

[23] H. Ratiney, M. Sdika, Y. Coenradie, S. Cavassila, D. v. Ormondt, and D. Graveron-Demilly, "Time-domain semiparametric estimation based on a metabolite basis set," $N M R$ in Biomedicine, vol. 18, no. 1, pp. 1-13, 2005.

[24] S. F. Cotter, B. D. Rao, K. Engan, and K. Kreutz-Delgado, "Sparse solutions to linear inverse problems with multiple measurement vectors," IEEE Transactions on Signal Processing, vol. 53, no. 7, pp. 2477-2488, 2005.

[25] J. Chen and X. Huo, "Theoretical results on sparse representations of multiple-measurement vectors," IEEE Transactions on Signal processing, vol. 54, no. 12, pp. 4634-4643, 2006.

[26] S. Posse, R. Otazo, S. R. Dager, and J. Alger, "MR spectroscopic imaging: principles and recent advances.," Journal of magnetic resonance imaging : JMRI, vol. 37, no. 6, pp. 1301-1325, June 2013.

[27] S. Hu, M. Lustig, A. P. Chen, J. Crane, A. Kerr, D. A. Kelley, R. Hurd, J. Kurhanewicz, S. J. Nelson, and J. M. Pauly, "Compressed sensing for resolution enhancement of hyperpolarized 13C flyback 3D-MRSI," Journal of magnetic resonance, vol. 192, no. 2, pp. 258-264, 2008.

[28] J. A. Fessler, "Image reconstruction toolbox," https://web.eecs.umich.edu/ fessler/code.

[29] J. A. Fessler and B. P. Sutton, "Nonuniform fast fourier transforms using min-max interpolation," IEEE transactions on signal processing, vol. 51, no. 2, pp. 560-574, 2003.

[30] W. Bogner, R. Otazo, and A. Henning, "Accelerated mr spectroscopic imaging - a review of current and emerging techniques," NMR in Biomedicine, vol. 34, no. 5, pp. e4314, 2021, e4314 NBM-19-0277.R2.

[31] J. Ziniel and P. Schniter, "Efficient high-dimensional inference in the multiple measurement vector problem," IEEE Transactions on Signal Processing, vol. 61, no. 2, pp. 340-354, 2012.

[32] N. Vaswani and W. Lu, "Modified-cs: Modifying compressive sensing for problems with partially known support," IEEE Transactions on Signal Processing, vol. 58, no. 9, pp. 45954607, 2010.

[33] J. W. Phillips, R. M. Leahy, and J. C. Mosher, "Megbased imaging of focal neuronal current sources," IEEE Transactions on Medical Imaging, vol. 16, no. 3, pp. 338348, 1997.

[34] I. F. Gorodnitsky, J. S. George, and B. D. Rao, "Neuromagnetic source imaging with focuss: a recursive weighted minimum norm algorithm," Electroencephalography and clinical Neurophysiology, vol. 95, no. 4, pp. 231-251, 1995. 